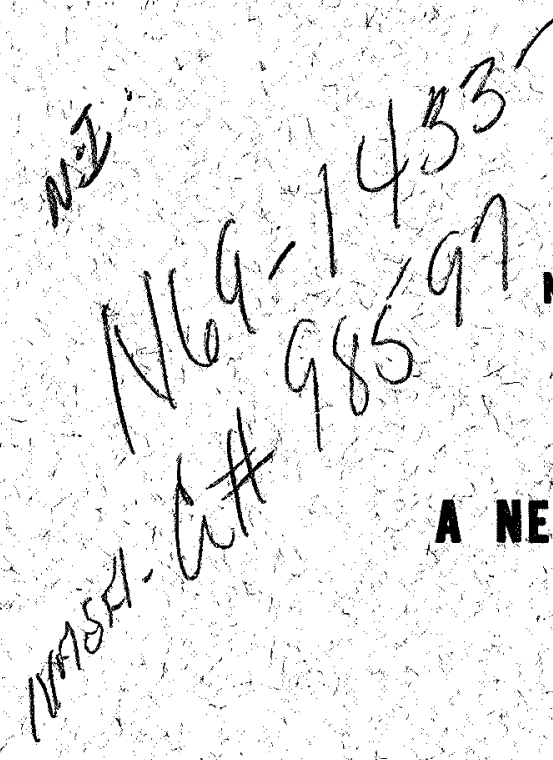

Contract N00014-67-A-0298-0006 MR-372-012

MATIOMAL AEROHAUTICS AMD SPACE ADMINISTRATIOH

Grant NGR 22.007.068

\title{
NEW NECESSARY CONDITION OF OPTIMALITY FOR SINGULAR CONTROL PROBLEMS
}
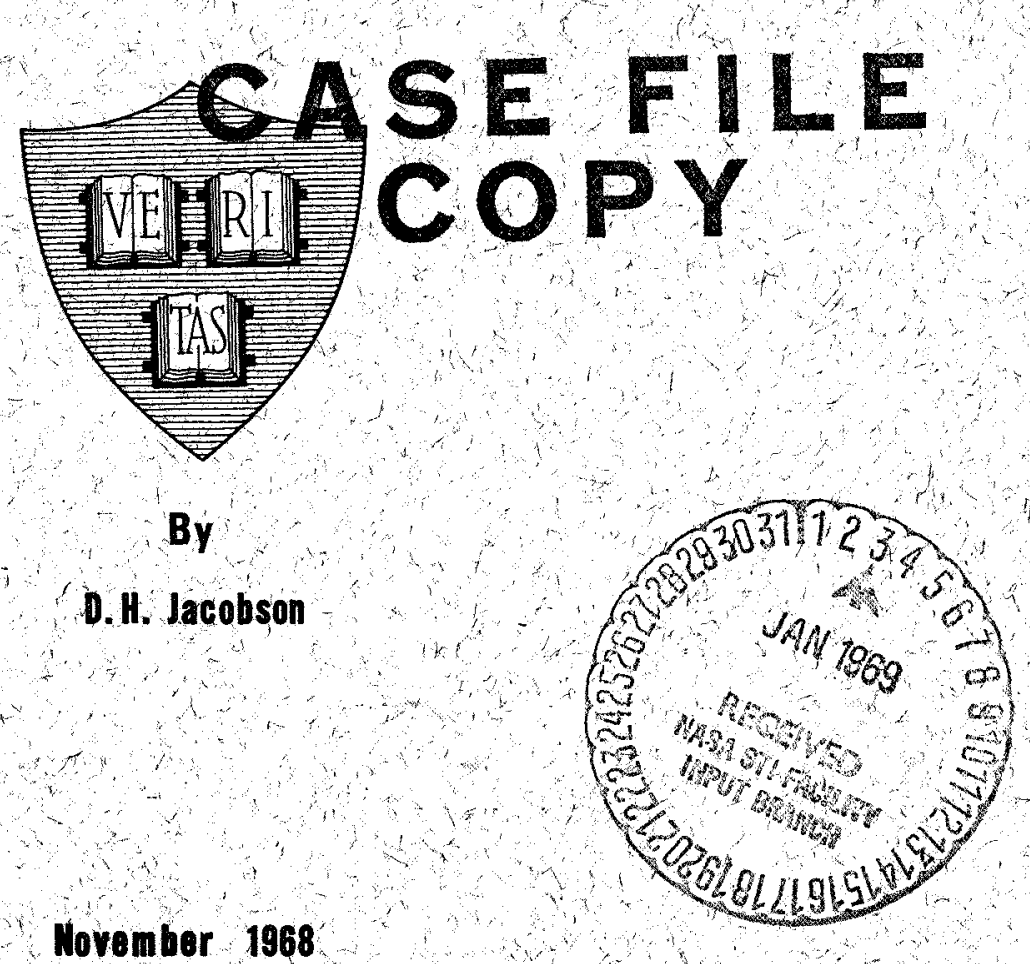

Technical Report Mo. 576

This document has been approved for pulfic roleast
and sale; its distribution is anlimited. Reproduction in whole or in mert is nermitted he the i S Fouernment.

Division of Engineering and Applied Physics

Harvard University - Cambridge. Massachusetts 
Office of Naval Research

Contract N00014-67-A-0298-0006

NR $-372-012$

National Aeronautics and Space Administration

Grant NGR 22-007-068

\begin{abstract}
A NEW NECESSARY CONDITION OF OPTIMALITY FOR SINGULAR CONTROL PROBLEMS

By

D. H. Jacobson
\end{abstract}

Technical Report No. 576

This document has been approved for public release and sale; its distribution is unlimited. Reproduction in whole or in part is permitted by the U. S. Government.

November 1968

The research reported in this document was made possible through support extended the Division of Engineering and Applied Physics, Harvard University by the U. S. Army Research Office, the U. S. Air Force Office of Scientific Research and the U. S. Office of: Naval Research under the Joint Services Electronics Program by Contracts N00014-67-A-0298-0006, 0005, and 0008 and by the National Aeronautics and Space Administration under Grant NGR 22-007-068.

Division of Engineering and Applied Physics

Harvard University Cambridge, Massachusetts 


\title{
A NEW NECESSARY CONDITION OF OPTIMALITY FOR SINGULAR CONTROL PROBLEMS
}

\author{
By \\ D. H. Jacobson \\ Division of Engineering and Applied Physics \\ Harvard University · Cambridge, Massachusetts
}

\begin{abstract}
$\underline{\text { ABSTRACT }}$
A variation in the form of a rectangular pulse of short duration, is introduced into the singular control function. The technique of Differential Dynamic Programming is used to obtain an expression for the change in cost produced by the control variation, and a new necessary condition of optimality is deduced by requiring that this change in cost be non-negative. When terminal equality constraints are present the control variation takes the form of a rectangular pulse followed by a 'special variation' which is chosen to keep the terminal equality constraints satisfied to first-order. Simple control problems are used to illustrate the non-equivalence of the new necessary condition and the generalized Legendre-Clebsch condition.
\end{abstract}




\section{Introduction}

Necessary conditions of optimality for non-singular, unconstrained, control problems are well known. When control and state variable constraints are present, the situation is more complex, but recent research [1]-[7] indicates that many of the subtleties of this class of problems are now uncovered. In the classical Calculus of Variations literature, little space is devoted to the analysis of singular variational problems. Recently, interest has been aroused in singular optimal control problems $[8]-[19]^{\dagger}$, owing to the appearance of such problems in, for example, the aerospace field and the chemical industry. Kelley discovered [20], and Robbins [21], Tait [22] and Kelley et al [23] generalized, a new necessary condition of optimality for singular arcs. The condition, known as the generalized Legendre-Clebsch condition, has, in a number of cases, proved useful [16], [18], [23] in eliminating some stationary arcs from the class of candidate arcs for minimizing solutions. The generalized Legendre-Clebsch condition is proved using special control variations. In this paper, by the use of a different special control variation, an additional necessary condition of optimality is derived. $\neq$ The Differential Dynamic Programming approach, outlined in [7], [24]-[26], is used to calculate the expression for the change in cost produced by the introduction of the special variation. The new necessary condition is deduced from this expression. Control problems without terminal constraints are considered first. For this class of problems, the special control variation is a

† Many additional references are given in [11], [21] and [23].

\# Some control problems are described which illustrate the necessity of the new condition in cases where the generalized Legendre-Clebsch condition is satisfied. 
rectangular pulse. With terminal constraints present, the rectangular pulse is followed by a control variation which is designed to keep the terminal constraints satisfied to first-order.

\section{Preliminaries}

Consider the class of control problems where the dynamical system is described by the differential equations:

$$
\dot{x}=f(x, u, t) \quad ; \quad x\left(t_{0}\right)=x_{0}
$$

where

$$
f(x, u, t) \equiv f_{1}(x, t)+f_{u}(x, t) u
$$

The performance of the system is measured by the cost functional

$$
V\left(x_{0}, t_{0}\right)=\int_{t_{0}}^{t_{f}} L(x, t) d t+F\left(x\left(t_{f}\right), t_{f}\right)
$$

and the terminal state must satisfy

$$
\psi\left(x\left(t_{f}\right), t_{f}\right)=0
$$

The control $u$ is required to satisfy the constraint

$$
|u(t)| \leqslant 1 ; t \in\left[t_{o}, t_{f}\right] .
$$

Here, $\mathbf{x}$ is an $n$-dimensional state vector, and $u$ is a scalar control. $f_{1}$ and $f_{u}$ are $n$-dimensional vector functions of $x$ at time $t$, and $L$ and $F$ are scalar functions. $\psi$ is an $s$-dimensional column vector function of $x\left(t_{f}\right)$ at $t_{f}$. The final time is assumed to be given explicitly. The functions $f, L$ and $F$ are assumed to be three times continuously differentiable in each argument.

The control problem is: determine the control function $u(\cdot)$ to satisfy (5) and (4) and minimize the cost $V\left(x_{0}, t_{0}\right)$. In general the optimal control function $u(\cdot)$ will consist of bang-bang sub-arcs and singular 
sub-arcs. A bang-bang arc is one along which strict equality holds in (5), except at a finite number of 'switch times' where the control u changes sign. A singular arc is one along which the control is interior to the constraint set; that is, strict inequality holds in (5).

\section{Necessary Conditions of Optimality}

It can be shown, for the case where terminal constraints are absent, that along a singular arc the following necessary conditions of optimality hold:

$$
\begin{aligned}
& -\dot{\bar{v}}_{x}=H_{x}\left(\bar{x}, \bar{u}, \bar{v}_{x}, t\right) \quad ; \quad \bar{v}_{x}\left(t_{f}\right)=F_{x}\left(\bar{x}\left(t_{f}\right), t_{f}\right) \\
& H_{u}\left(\bar{x}, \bar{v}_{x}, t\right)=0
\end{aligned}
$$

where

$$
H\left(x, u, V_{x}^{\prime}, t\right)=L(x, t)+\left\langle V_{x}, f(x, u, t)\right\rangle
$$

and $\bar{x}(\cdot), \bar{u}(\cdot)$ denote the candidate state and control functions. The derivative $\bar{V}_{x}(\bar{x}, t)$ is the first partial derivative ${ }^{\neq}$of $\bar{V}-$ the cost produced by the control function $\bar{u}(\cdot)$. Here, $\bar{V}_{x}$ can be identified with Pontryagin's adjoint variable. Note that $\bar{V}_{x}$ is not the first partial derivative $V_{x}^{O}$ of the optimal cost $\mathrm{V}^{\mathrm{O}}$ which is obtained when optimal feedback control is used.

Along a singular arc, Kelley et al [20], Robbins [21] and Tait [22] prove that an additional necessary condition of optimality is:

$$
(-1)^{p} \frac{\partial}{\partial u}\left[\frac{d^{2 p}}{d t^{2 p}} H_{u}\left(\bar{x}, \bar{v}_{x}, t\right)\right] \geqslant 0
$$

where the $2 \mathrm{p}$-th time derivative of $\mathrm{H}_{\mathrm{u}}$ is the first to contain explicitly the control u. Inequality (9) is known as the generalized Legendre-Clebsch condition.

f From this point on, 'arc' and 'sub-arc' are used synonymously.

$\neq \bar{V}_{x}$ is determined by changing $x$ but keeping the control function unchanged at $\vec{u}(\cdot)$. 


\section{Expression for Change in Cost when Control Variation is Present:}

\section{Terminal State Unconstrained.}

If a control function $\bar{u}(\cdot)+\delta u(\cdot)$ is applied to the system, then a trajectory $\bar{x}(\cdot)+\delta_{x}(\cdot)$ is produced. At time $t, v\left(\bar{x}+\delta_{x}, t\right)$ is the cost to go, from $t$ to the final time $t_{f}$, when starting in state $\bar{x}(t)+\delta_{x}(t)$ and using controls $\bar{u}(\cdot)+\delta u(\cdot)$. Let us assume that the cost can be expanded in a Taylor series about $\bar{x}, t$ :

$$
\begin{gathered}
\mathrm{V}\left(\bar{x}+\delta_{x}, t\right)=V(\bar{x}, t)+\left\langle v_{x}(\bar{x}, t), \delta_{x}\right\rangle+\frac{1}{2}\left\langle\delta_{x}, v_{x x}(\bar{x}, t) \delta_{x}\right\rangle \\
+ \text { higher-order terms } .
\end{gathered}
$$

The partial derivatives in (10) are obtained by changing $\mathrm{x}$ but keeping the control function fixed ${ }^{\dagger}$ at $\bar{u}(\cdot)+\delta u(\cdot)$. V $(\bar{x}, t)--$ the cost to go from $t$ to $t_{f}$ when starting in state $\bar{x}(t)$ and using controls $\bar{u}(\cdot)+\delta u(\cdot)--$ can be written as

$$
V(\bar{x}, t)=\bar{V}(\bar{x}, t)+a(\bar{x}, t)
$$

where al $(\overrightarrow{\mathrm{x}}, t)$, is the change in cost, when starting at time $t$ in state $\bar{x}(t)$, produced by the variation $\delta u(\tau), \tau \in\left[t, t_{f}\right]$.

Using (11) in (10):

$$
\begin{aligned}
V\left(\bar{x}+\delta_{x}, t\right)= & \bar{V}(\bar{x}, t)+a(\bar{x}, t)+\left\langle V_{x}(\bar{x}, t), \delta_{x}\right\rangle+\frac{1}{2}\left\langle\delta_{x}, V_{x x}(\bar{x}, t) \delta_{x}\right\rangle \\
& + \text { higher-order terms. }
\end{aligned}
$$

From (3) it is clear that

$$
\dot{\mathrm{V}}\left(\overline{\mathrm{x}}+\delta_{\mathrm{x}}, t\right)=-L\left(\overline{\mathrm{x}}+\delta_{\mathrm{x}, t}\right)
$$

whence,

$$
-\frac{\partial V}{\partial t}\left(\bar{x}+\delta_{x}, t\right)=L\left(\bar{x}+\delta_{x}, t\right)+\left\langle V_{x}\left(\bar{x}+\delta_{x}, t\right), f\left(\bar{x}+\delta_{x}, \bar{u}+\delta_{u}, t\right)\right\rangle .
$$

+ c.f. Section 3 . 
Substituting (12) into (14) and expanding $L$ and $f$ in Taylor series about $\bar{x}$, we obtain:

$$
\begin{aligned}
& -\frac{\partial \bar{V}}{\partial t}-\frac{\partial a}{\partial t}-\left\langle\frac{\partial V}{\partial t}, \delta_{x}\right\rangle-\frac{1}{2}\left\langle\delta_{x}, \frac{\partial V_{x x}}{\partial t} \delta_{x}\right\rangle+\text { higher-order terms }= \\
& H\left(\bar{x}, \bar{u}+\delta_{u}, V_{x}, t\right)+\left\langle H_{x}+V_{x x} f, \delta_{x}\right\rangle \\
& +\frac{1}{2}\left\langle\delta_{x},\left(H_{x x}+f_{x}^{T} V_{x x}+V_{x x} f_{x}+\frac{1}{2} f^{T} V_{x x x}+\frac{1}{2} V_{x x x} f\right) \delta_{x}\right\rangle \\
& + \text { higher-order terms. }
\end{aligned}
$$

All derivatives in (15) are evaluated at $\bar{x}, \bar{u}+\delta u, V$, t.

Since equality holds for all $\delta_{x}$, we equate coefficients to obtain:

$$
\begin{aligned}
& -\frac{\partial \bar{V}}{\partial t}-\frac{\partial a}{\partial t}=H\left(\bar{x}, \bar{u}+\delta u, v_{x}, t\right) \\
& -\frac{\partial V_{x}}{\partial t}=H_{x}\left(\bar{x}, \bar{u}+\delta u_{x}, t\right)+V_{x x} f(\bar{x}, \bar{u}+\delta u, t) \\
& -\frac{\partial V_{x x}}{\partial t}=H_{x x}\left(\bar{x}, \bar{u}+\delta u, V_{x}, t\right)+f_{x}^{T}\left(\bar{x}, \bar{u}+\delta_{u, t}\right) V_{x x}+V_{x x_{x}} f_{x}(\bar{x}, \bar{u}+\delta u, t) \\
& +\frac{1}{2} V_{x x x} f(\bar{x}, \bar{u}+\delta u, t)+\frac{1}{2} f^{T}(\bar{x}, \bar{u}+\delta u, t) V_{x x x}
\end{aligned}
$$

The higher-order equations are not presented.

Now,

$$
\frac{d}{d t}(\bar{V}+a)=\frac{d}{d t} V=\frac{\partial V}{\partial t}+\left\langle V v_{x}, f(\bar{x}, \bar{u}, t)\right\rangle
$$

Therefore,

$$
\frac{d}{d t}(\bar{v}+a)=\frac{\partial}{\partial t}(\bar{v}+a)+\left\langle v_{x}, f(\bar{x}, \bar{u}, t)\right\rangle
$$

and

$$
\begin{aligned}
& \dot{V}_{x}=\frac{\partial V_{x}}{\partial t}+V_{x x} f(\bar{x}, \bar{u}, t) \\
& \dot{V}_{x x}=\frac{\partial V_{x x}}{\partial t}+\frac{1}{2} V_{x x x} f(\bar{x}, \bar{u}, t)+\frac{1}{2} f^{T}(\bar{x}, \bar{u}, t) V_{x x x}
\end{aligned}
$$


Using (17) in (16), the following equations result:

$$
\begin{aligned}
& -\dot{\mathrm{a}}=\mathrm{H}-\mathrm{H}\left(\overline{\mathrm{x}}, \overline{\mathrm{u}}, \mathrm{V}_{x}, t\right) \\
& -\dot{\mathrm{V}}_{x}=\mathrm{H}_{x}+\mathrm{V}_{x x}(f-f(\bar{x}, \bar{u}, t)) \\
& -\dot{V}_{x x}=H_{x x}+f_{x}^{T} V_{x x}+V_{x x_{x}}+\frac{1}{2} V_{x x x}(f-f(\bar{x}, \bar{u}, t)) \\
& \quad+\frac{1}{2}(f-f(\bar{x}, \bar{u}, t))^{T_{V_{x x x}}}
\end{aligned}
$$

where, unless otherwise specified, all quantities are evaluated at $\bar{x}, \bar{u}+\delta u$, $V_{x}$, t. Using the special structure of $f$, Eq. (2), equations (18) become:

$$
\begin{aligned}
& -\dot{a}=H_{u} \delta_{u} \\
& -\dot{\mathrm{V}}_{\mathrm{x}}=\mathrm{H}_{\mathrm{x}}+\left(\mathrm{H}_{\mathrm{xu}}+\mathrm{V}_{\mathrm{xx}} f_{\mathrm{u}}\right) \delta_{\mathrm{u}} \\
& -\dot{\mathrm{V}}_{\mathrm{xx}}=\mathrm{H}_{\mathrm{xx}}+\mathrm{f}_{\mathrm{x}}^{\mathrm{T}} \mathrm{V}_{\mathrm{xx}}+\mathrm{V}_{\mathrm{xx} \mathrm{f}_{\mathrm{x}}}+\left(\mathrm{H}_{\mathrm{xxu}}+\mathrm{f}_{\mathrm{xu}}^{\mathrm{T}} \mathrm{V}_{\mathrm{xx}}+\mathrm{V}_{\mathrm{xx} x \mathrm{fu}}\right. \\
& +\frac{1}{2} V_{x x x} f u+\frac{1}{2} f_{u}^{T} V_{x x x} j \delta_{u}
\end{aligned}
$$

In (19), all quantities are now evaluated at $\bar{x}, \bar{u}, v_{x}$, t. Boundary conditions for Eqs. (19) are, clearly,

$$
\begin{aligned}
& a\left(t_{f}\right)=0 \\
& v_{x}\left(t_{f}\right)=F_{x}\left(\bar{x}, t_{f}\right) \\
& V_{x x}\left(t_{f}\right)=F_{x x}\left(\bar{x}, t_{f}\right)
\end{aligned}
$$

The change in cost owing to the presence of a control variation $\delta u(\tau)$;

$\tau \in\left[\mathrm{t}_{1}, \mathrm{t}_{2}\right], \mathrm{t}_{2}>\mathrm{t}_{1}$, is given by

$$
a\left(t_{1}\right)=a\left(t_{2}\right)+\int_{t_{2}}^{t_{1}} \dot{a}(t) d t
$$

\section{New Necessary Condition: Unconstrained Terminal State}

A singular arc is assumed to lie in an interval $\left[\mathrm{t}_{\mathrm{a}}, \mathrm{t}_{\mathrm{b}}\right]$. A control variation in the form of a rectangular pulse of height $\eta$ and duration $T$ is introduced in an interval $\left[t_{1}, t_{2}\right]$ where: 


$$
t_{a}<t_{i}<t_{b} \quad ; \quad i=1,2, t_{2}>t_{1}
$$

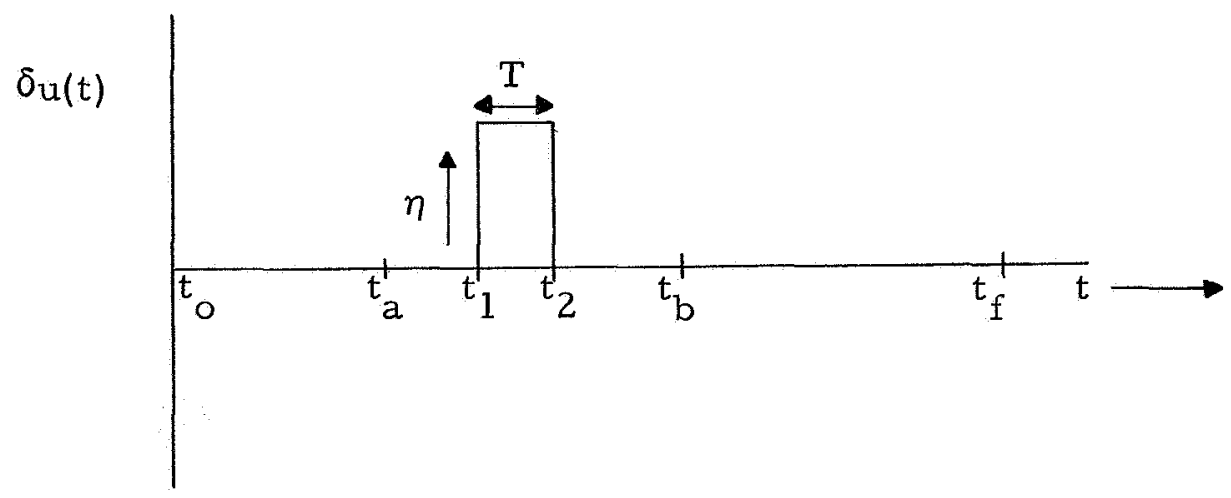

Figure 1

The change in cost produced by this variation is given by

$$
a\left(t_{1}\right)=\int_{t_{2}}^{t_{1}} \dot{a} d t+a\left(t_{2}\right)=\int_{t_{2}}^{t_{1}}-H_{u} \delta u d t+a\left(t_{2}\right)
$$

where $H_{u}$ is evaluated at $\bar{x}, V_{x}$, t. Expanding the integral in a Taylor series in $T$, the expression for the change in cost becomes:

$$
a\left(t_{1}\right)=\left.H_{u} \delta u\right|_{t_{2}} T-\left.\frac{1}{2} \frac{d}{d t}\left[H_{u} \delta u\right]\right|_{t_{2}} T^{2}+\ldots+a\left(t_{2}\right) .
$$

At time $t_{2}$, one has

$$
\begin{aligned}
& a\left(t_{2}\right)=0 \\
& v_{x}\left(t_{2}\right)=\bar{v}_{x}\left(t_{2}\right) \\
& v_{x x}\left(t_{2}\right)=\bar{v}_{x x}\left(t_{2}\right)
\end{aligned}
$$

where $\bar{V}_{x}\left(t_{2}\right)$ and $\bar{V}_{x x}\left(t_{2}\right)$ are computed using (19) and (20) with $\delta u(t)=0$, $t \in\left(t_{2}, t_{f}\right]$.

+ Note that in (24) quantities are evaluated at the time instant immediately prior to time $t_{2}$. 
Since $\bar{x}\left(t_{2}\right)$ is on the singular arc, $H_{u}\left(\bar{x}, \bar{v}_{x}, t_{2}\right)=0$. Thus, the first non-zero term in expansion (24) is the $T^{2}$ one. We have that:

$$
\left.\frac{\mathrm{d}}{\mathrm{dt}}\left[\begin{array}{ll}
\mathrm{H}_{\mathrm{u}} & \delta \mathrm{u}
\end{array}\right]\right|_{\mathrm{t}_{2}}=\left.\dot{\mathrm{H}}_{\mathrm{u}} \delta \mathrm{u}\right|_{\mathrm{t}_{2}}+\left.\mathrm{H}_{\mathrm{u}} \delta \dot{\mathrm{u}}\right|_{\mathrm{t}_{2}}=\left.\dot{\mathrm{H}}_{\mathrm{u}}\left(\overline{\mathrm{x}}, \mathrm{v}_{\mathrm{x}}, \mathrm{t}\right)\right|_{\mathrm{t}_{2}} \eta
$$

From (19), (20),

$$
\left.\dot{H}_{u}\left(\bar{x}, v_{x}, t\right)\right|_{t_{2}}=\left.\left\{\dot{f}_{u}^{T} v_{x}+f_{u}^{T}\left[-H_{x}-\left(H_{x u}+v_{x x} f_{u}\right) \eta\right]\right\}\right|_{t_{2}}
$$

The first two terms in (27) sum to zero. ${ }^{\dagger} \mathrm{Using}(27)$ and (26) in (24), the change in cost is

$$
\begin{aligned}
a\left(t_{1}\right)= & \frac{1}{2} f_{u}^{T}\left(\bar{x}, t_{2}\right)\left[H_{x u}\left(\bar{x}, \bar{v}_{x}, t_{2}\right)+\bar{v}_{x x u}\left(\bar{x}, t_{2}\right)\right] \eta^{2} \mathrm{~T}^{2} \\
& + \text { higher-order terms }
\end{aligned}
$$

For the singular arc to be a condidate as a minimizing arc, it is necessary that the change in cost, owing to the presence of the control variation, be non-negative. From (28) this implies that

$$
f_{u}^{T}(\bar{x}, t)\left[H_{x u}\left(\bar{x}, \bar{u}, \bar{v}_{x}, t\right)+\bar{v}_{x x_{u}} f(\bar{x}, t)\right] \geqslant 0
$$

where

$$
\begin{aligned}
& -\dot{\bar{V}}_{x}=H_{x}\left(\bar{x}, \bar{u}, v_{x}, t\right) \\
& -\dot{\bar{V}}_{x x}=H_{x x}\left(\bar{x}, \bar{u}_{,} v_{x}, t\right)+f_{x}^{T}(\bar{x}, \bar{u}, t) \bar{V}_{x x}+\bar{V}_{x x} f_{x}(\bar{x}, \bar{u}, t)
\end{aligned}
$$

and

$$
\begin{aligned}
& \bar{V}_{x}\left(t_{f}\right)=F_{x}\left(\bar{x}\left(t_{f}\right), t_{f}\right) \\
& \bar{V}_{x x}\left(t_{f}\right)=F_{x x}\left(\bar{x}\left(t_{f}\right), t_{f}\right)
\end{aligned}
$$

Inequality (29) is the new necessary condition of optimality for singular control problems with unconstrained terminal states.

$+\dot{H}_{u}\left(\bar{x}, \bar{v}_{x}, t_{2}\right)=0=\dot{f}_{u}^{T} \bar{v}_{x}-f_{u}^{T} H_{x}\left(\bar{x}, \bar{u}, \bar{v}_{x}, t_{2}\right)$ 


\section{Examples}

1. Consider the following scalar control problem:

$$
\begin{aligned}
& \dot{x}=u \quad ; \quad x(0)=1 \\
& v(1,0)=\int_{0}^{2} x^{2} d t \\
& |u| \leqslant 1
\end{aligned}
$$

The optimal control is

$$
\begin{array}{lll}
\bar{u}(t)=-1 & , & t \in[0,1] \\
\bar{u}(t)=0 & , \quad t \in(1,2]
\end{array}
$$

The arc in $x, t$ space along which $u(t)$ is zero, is singular.

For the above problem we have that

$$
\begin{aligned}
& H\left(x, u, v_{x}, t\right)=x^{2}+V_{x} u \\
& H_{u}\left(x, V_{x}, t\right)=V_{x} \\
& -\overline{\bar{V}}_{x}=2 \bar{x} ; \quad \bar{V}_{x}\left(t_{f}\right)=0 \\
& -\dot{\bar{V}}_{x x}=2 ; \quad \bar{V}_{x x}\left(t_{f}\right)=0 \\
& \dot{H}_{u}=-2 x
\end{aligned}
$$

and

$$
\ddot{H}_{u}=-2 u
$$

whence

$$
\frac{\partial}{\partial u} \ddot{H}_{u}=-2
$$

so that the generalized Legendre-Clebsch condition is satisfied.

It is clear that

$$
f_{u}^{T}\left(H_{x u}+\bar{V}_{x x} f_{u}\right)=\bar{V}_{x x}
$$


and from (36)

$$
\overline{\mathrm{V}}_{\mathrm{xx}}(\tau)=2 \tau
$$

where

$$
\tau=2-\mathrm{t} .
$$

From (40),

$$
\overline{\mathrm{V}}_{\mathrm{xx}}(\tau) \geqslant 0 \quad \forall \tau \in[1,2]
$$

so that the new necessary condition is satisfied.

Let us consider now the following cost functional

$$
V(1,0)=\int_{0}^{2} x^{2} d t-s x^{2}\left(t_{f}\right)
$$

where $\mathbf{S}$ is positive. The control program (34) is a stationary solution for this cost functional because the first-order necessary conditions of optimality are satisfied. Moreover, the generalized Legendre-Clebsch condition is satisfied. However, the differential equation for $\bar{V}_{x x}$ is

$$
-\dot{\overline{\mathrm{V}}}_{\mathrm{xx}}=2 ; \overline{\mathrm{V}}_{\mathrm{xx}}\left(\mathrm{t}_{\mathrm{f}}\right)=-\mathrm{S}
$$

and hence

$$
\overline{\mathrm{V}}_{\mathrm{xx}}(\tau)=-\mathrm{S}+2 \tau
$$

Since $S$ is positive, the new necessary condition is violated for $\tau$ sufficiently small. It can be verified directly that, for $S>\frac{1}{3}$, a control function

$$
\begin{array}{ll}
u(t)=-1, & t \in[0,1] \\
u(t)=\epsilon, & t \in(1,2]
\end{array}
$$

produces a cost lower than that resulting from the use of the control program (34), confirming the non-optimality of that control program.

2. Consider the second-order control problem:

$$
\begin{aligned}
& \dot{x}_{1}=x_{2} \quad ; \quad x_{1}(0)=0 \\
& \dot{x}_{2}=u, \quad ; \quad x_{2}(0)=1
\end{aligned}
$$




$$
\begin{aligned}
& V\left(x_{0}, 0\right)=\frac{1}{2} \int_{0}^{\frac{3 \pi}{2}}\left(x_{1}^{2}+x_{2}^{2}\right) d t \\
& |u| \leqslant 1
\end{aligned}
$$

Here,

$$
\begin{array}{lll}
-\dot{\overline{\mathrm{V}}}_{\mathrm{x}_{1}}=\overline{\mathrm{x}}_{1} & ; & \overline{\mathrm{v}}_{\mathrm{x}_{1}}\left(\mathrm{t}_{\mathrm{f}}\right)=0 \\
-\dot{\overline{\mathrm{V}}}_{\mathrm{x}_{2}}=\overline{\mathrm{x}}_{2}+\overline{\mathrm{V}}_{\mathrm{x}_{1}} \quad ; & \overline{\mathrm{v}}_{\mathrm{x}_{2}}\left(\mathrm{t}_{\mathrm{f}}\right)=0
\end{array}
$$

and

$$
\begin{aligned}
& \mathrm{H}_{\mathrm{u}}=\mathrm{V}_{\mathrm{x}_{2}} \\
& \dot{\mathrm{H}}_{\mathrm{u}}=-\mathrm{x}_{2}-\mathrm{v}_{\mathrm{x}_{1}} \\
& \ddot{\mathrm{H}}_{\mathrm{u}}=-\mathrm{u}-\mathrm{x}_{1}
\end{aligned}
$$

so that the generalized Legendre-Clebsch condition is satisfied. The expression $f_{u}^{T}\left(H_{x u}+\bar{V}_{x x^{\prime} u} f^{\prime}\right)$ is equal to $\bar{V}_{x_{2} x_{2}}$, and

$$
\overline{\mathrm{v}}_{\mathrm{x}_{2} \mathrm{x}_{2}}(\tau)=\tau+\frac{1}{3} \tau^{3}
$$

so that the new necessary condition is satisfied. It can be verified [9] that this problem has a stationary solution which exhibits a singular arc. Moreover, the stationary solution is minimizing.

Consider now the following cost functional:

$$
\mathrm{V}\left(\mathrm{x}_{\mathrm{o}}, 0\right)=\frac{1}{2} \int_{0}^{\frac{3 \pi}{2}}\left(-\mathrm{x}_{1}^{2}+\mathrm{x}_{2}^{2}\right) \mathrm{dt}
$$

Here,

$$
\begin{aligned}
& -\dot{\mathrm{V}}_{\mathrm{x}_{1}}=-\mathrm{x}_{1} \\
& -\dot{\mathrm{V}}_{\mathrm{x}_{2}}=\mathrm{x}_{2}+\mathrm{v}_{\mathrm{x}_{1}}
\end{aligned}
$$


and

$$
\begin{aligned}
& H_{u}=V_{x_{2}} \\
& \dot{H}_{u}=-x_{2}-V_{x_{1}} \\
& \ddot{H}_{u}=-u-x_{1}
\end{aligned}
$$

so that the generalized Legendre-Clebsch condition is satisfied.

$$
\begin{aligned}
& \text { It is easy to see that } \\
& \bar{x}_{1}(t)=\sin t \\
& \bar{x}_{2}(t)=\cos t
\end{aligned}
$$

is a singular.solution, and the cost functional value corresponding to this trajectory is zero.

One can verify that the equation for $\bar{V}_{x_{2} x_{2}}(\tau)$ is

$$
\overline{\mathrm{V}}_{\mathrm{x}_{2} \mathrm{x}_{2}}(\tau)=\tau-\frac{1}{3} \tau^{3}
$$

which is negative for $\tau>\sqrt{3}$ : that is, the new necessary condition is violated for $\tau>\sqrt{3}$. The control function corresponding to the trajectory (56) is,

$$
\bar{u}(t)=-\sin t \quad .
$$

Consider the control function

$$
u(t)=\bar{u}(t)+\epsilon
$$

where is a constant. Then, the resulting trajectory is

$$
\begin{aligned}
& \bar{x}_{2}(t)+\delta x_{2}(t)=\cos t+\epsilon t \\
& \bar{x}_{1}(t)+\delta_{x_{1}}(t)=\sin t+\frac{1}{2} \epsilon^{2}
\end{aligned}
$$

The integrand in (53), corresponding to trajectory (60) is:

$$
-\left(\sin ^{2} t+\epsilon t^{2} \sin t+\frac{\epsilon^{2}}{4} t^{4}\right)+\left(\cos ^{2} t+2 \epsilon t \cos t+\epsilon^{2} t^{2}\right) \text {. }
$$


Using (61) in (53),

$$
\begin{aligned}
V\left(x_{0}, 0\right) & =\frac{1}{2} \int_{0}^{2 \pi}\left[\cos ^{2} t-\sin ^{2} t+\epsilon\left(2 t \cos t-t^{2} \sin t\right)+\epsilon^{2}\left(-\frac{t^{4}}{4}+t^{2}\right)\right] d t \\
& =\frac{1}{2}\left[\frac{1}{2} \sin 2 t+\epsilon^{2} \cos t+\epsilon^{2}\left(-\frac{t^{5}}{20}+\frac{t^{3}}{3}\right)\right]_{0}^{3 \pi / 2} \\
& =-40.7 \epsilon^{2}
\end{aligned}
$$

which is negative, confirming the non-optimality of the singular trajectory.

The above examples illustrate the necessity of the new condition of optimality. Also demonstrated is the non-equivalence of the new condition and the generalized Legendre-Clebsch condition.

\section{Adjoining Terminal Constraint}

Here we consider the case where equality (4) is present. The equality constraint can be adjoined to the cost functional by a vector $b$ of Lagrange multipliers, in the following way.

$$
V^{*}\left(x_{0}, b, t_{0}\right)=\int_{t_{0}}^{t_{f}} L(x, t) d t+F\left(x\left(t_{f}\right), t_{f}\right)+\left\langle b, \psi\left(x\left(t_{f}\right), t_{f}\right)\right\rangle
$$

Assume that $\bar{x}(\cdot), \bar{b}$ and $\bar{u}(\cdot)$ are stationary solutions of $(64)$; the following necessary conditions are satisfied along a singular arc:

$$
\begin{aligned}
& -\dot{\bar{V}}_{x}^{* *}=H_{x}\left(\bar{x}, \bar{u}, \bar{V}_{x}^{*}, t\right) \quad ; \quad \bar{V}_{x}^{*}\left(t_{f}\right)=F_{x}\left(\bar{x}, t_{f}\right)+\psi_{x}^{T}\left(\bar{x}, t_{f}\right) \bar{b} \\
& H_{u}\left(\bar{x}, \vec{V}_{x}^{*}, t\right)=0 .
\end{aligned}
$$

If $\mathrm{V}^{* k}\left(\mathrm{x}_{0}, \overline{\mathrm{b}}, \mathrm{t}_{\mathrm{o}}\right)$ has an unconstrained minimum with respect to $u(\cdot)$ at $\overline{\mathrm{u}}(\cdot)$, the following condition must hold along the singular arc:

$$
f_{u}^{T}(\bar{x}, t)\left[H_{x u}\left(\bar{x}, \bar{V}_{x}^{*}, t\right)+\bar{V}_{x x_{u}^{*}}^{*}(\bar{x}, t)\right] \geqslant 0
$$


where

$$
-\dot{\bar{V}}_{x x}^{*}=H_{x x}+f_{x}^{T} \bar{v}_{x x}^{*}+\bar{v}_{x x x}^{*} f_{x x} ; \bar{v}_{x x}^{*}\left(t_{f}\right)=F_{x x}\left(\bar{x}, t_{f}\right)+\bar{b} \psi_{x x}\left(\bar{x}, t_{f}\right)
$$

Condition (66) follows from Section 5.

However, failure of condition (66) does not imply that $\bar{x}(\cdot), \bar{u}(\cdot)$ is not a minimizing solution for the constrained problem where equality (4) is enforced. This is so because a minimizing solution of the original constrained problem need only be a stationary solution of (64) for fixed $\mathrm{b}=\overline{\mathrm{b}}$. In order to determine whether $\overline{\mathrm{x}}(\cdot), \overline{\mathrm{u}}(\cdot)$ is a possible minimizing solution, one has to ensure that, on the introduction of a control variation, equality (4) remains satisfied.

\section{New Necessary Condition: Constrained Terminal State}

Let us assume, as in Section 5, that a control variation consisting of a rectangular pulse of duration $t_{2}-t_{1} \equiv T$ and height $\eta$ is introduced in the singular control interval $\left[t_{a}, t_{b}\right]$. A further control variation is now introduced in the interval $\left(t_{2}, t_{b}\right)$ in order to force equality (4) to remain satisfied. We shall assume the following form for the control variation in the interval $\left(t_{2}, t_{b}\right]$ :

$$
\delta u(\tau)=\beta(\tau) \sigma, \quad ; \quad \tau \in\left(t_{2}, t_{b}\right] .
$$

Here, $\beta(\tau)$ is a time varying, r-dimensional row vector and $\sigma$ is a constant $r$-dimensional vector. For $\delta_{\mathrm{X}}$ and $\delta_{\mathrm{u}}$ sufficiently small, the following equations are valid:

$$
\begin{array}{lll}
\delta \dot{x}(\tau)=f_{x}(\bar{x}, \bar{u}, \tau) \delta_{x}(\tau)+f_{u}(\bar{x}, \tau) \beta(\tau) \sigma & ; & \tau \in\left(t_{2}, t_{b}\right] \\
\delta \dot{x}(\tau)=f_{x}(\bar{x}, \bar{u}, \tau) \delta_{x}(\tau) & ; & \tau \in\left(t_{b}, t_{f}\right]
\end{array}
$$

where $\delta_{x}\left(t_{2}\right) \neq 0$ owing to the rectangular pulse variation prior to $\dot{t}_{2}$. In order that the control constraints (5) remain satisfied when (68) is used, 
it is assumed that $\bar{u}(\tau), \tau \in\left(t_{a}, t_{b}\right)$ is in the interior of the control constraint set. $t$

The solution of Eqs. (69) is:

$$
\delta_{x}(t)=\Phi\left(t, t_{2}\right) \delta_{x}\left(t_{2}\right)+\int_{t_{2}}^{t} \Phi(t, \tau) f_{u}(\tau) \beta(\tau) \sigma d \tau
$$

with

$$
\beta(\tau) \sigma \equiv 0 \quad \tau \in\left(t_{b}, t_{f}\right]
$$

where $\Phi(t, \tau)$ satisfies the differential equation

$$
\frac{\mathrm{d}}{\mathrm{dt}} \Phi(\mathrm{t}, \tau)=\mathrm{f}_{\mathrm{x}}(\overline{\mathrm{x}}, \overline{\mathrm{u}}, \mathrm{t}) \Phi(t, \tau) \quad ; \quad \Phi(\tau, \tau)=\mathrm{I}
$$

At $t=t_{f}$, we require, for $\delta_{x}\left(t_{f}\right)$ sufficiently small, that

$$
\psi_{x}\left(\bar{x}, t_{f}\right) \delta_{x}\left(t_{f}\right)=0
$$

Setting $t=t_{f}$ in (70), and using (72), we obtain

$$
0=\psi_{x}\left(\bar{x}, t_{f}\right) \Phi\left(t_{f}, t_{2}\right) \delta_{x}\left(t_{2}\right)+\psi_{x}\left(\bar{x}, t_{f}\right) \int_{t_{2}}^{t_{f}} \Phi\left(t_{f}, \tau\right) f_{u}(\tau) \beta(\tau) \sigma d \tau
$$

which, by $(70)$, is equivalent to

$$
0=\psi_{x}\left(\bar{x}, t_{f}\right) \Phi\left(t_{f}, t_{2}\right) \delta_{x}\left(t_{2}\right)+\psi_{x}\left(\bar{x}, t_{f}\right) \int_{t_{2}}^{t_{b}} \Phi\left(t_{f}, \tau\right) f_{u}(\tau) \beta(\tau) \sigma d \tau
$$

Let us choose

$$
\beta(\tau)=f_{u}^{T}(\tau) \Phi^{T}\left(t_{f}, \tau\right) \psi_{x}^{T}\left(\bar{x}, t_{f}\right)
$$

Using (75) in (74), we obtain

+ If the singular control and the non-singular 'bang' control are continuous at $t_{b}$, then (68) is used up until $t_{b}-\epsilon, \epsilon>0$ to ensure that the control constraints remain satisfied in the interval $\left(t_{2}, t_{b}\right)$. 
$\psi_{x}\left(\bar{x}, t_{f}\right)\left[\int_{t_{2}}^{t_{b}} \Phi\left(t_{f}, \tau\right) f_{u}(\tau) f_{u}^{T}(\tau) \Phi^{T}\left(t_{f}, \tau\right) d \tau\right] \psi_{x}^{T}\left(\bar{x}, t_{f}\right) \sigma=-\psi_{x}\left(\bar{x}, t_{f}\right) \Phi\left(t_{f}, t_{2}\right) \delta_{x}\left(t_{2}\right)$

Denoting the contents of the square brackets on the left-hand side of (76) by

$$
w\left(t_{2}, t_{b}\right)
$$

we obtain $t$

$$
\left.\begin{array}{rl}
\sigma & =-\left[\psi_{x}\left(\bar{x}, t_{f}\right) W\left(t_{2}, t_{b}\right) \psi_{x}^{T}\left(\bar{x}, t_{f}\right)\right]^{-1} \psi_{x}\left(\bar{x}, t_{f}\right) \Phi\left(t_{f}, t_{2}\right) \delta_{x}\left(t_{2}\right) \\
& \equiv \gamma \delta_{x}\left(t_{2}\right)
\end{array}\right\}
$$

We have, for $\delta_{x}\left(t_{2}\right)$ sufficiently small, i.e., for $\eta$ (or $\mathrm{T}$ ) sufficiently small, that if expressions (75) and (78) are used in (68), then equality is maintained to first-order. That is, the change in $\delta_{x}\left(t_{f}\right)$ is at most of order $\left[\delta_{x}\left(t_{2}\right)\right]^{2}$

For $\tau \epsilon\left(t_{b}, t_{f}\right]$ we have the same equations for $\bar{V}_{x}^{*}$ and $\bar{V}_{x x}^{*}$, namely

$$
\begin{aligned}
& \left.-\dot{\mathrm{V}}_{\mathrm{x}}^{* *}=\mathrm{H}_{\mathrm{x}}\left(\overline{\mathrm{x}}, \overline{\mathrm{u}}, \overline{\mathrm{V}}_{\mathrm{x}}^{*}, \mathrm{t}\right) \quad ; \quad \overline{\mathrm{V}}_{\mathrm{x}}^{*}\left(\mathrm{t}_{\mathrm{f}}\right)=\mathrm{F}_{\mathrm{x}}+\left.\psi_{\mathrm{x}}^{\mathrm{T}} \overline{\mathrm{b}}\right|_{\mathrm{t}_{\mathrm{f}}}\right) \\
& -\dot{\bar{V}}_{x x}^{*}=H_{x x}\left(\bar{x}, \bar{u}, \bar{V}_{x}^{*}, t\right)+f_{x}^{T}(\bar{x}, \bar{u}, t) \bar{V}_{x x}^{*}+\bar{V}_{x x}^{*} f_{x}(\bar{x}, \bar{u}, t) ; \\
& \bar{V}_{x x}^{*}\left(t_{f}\right)=F_{x x}+\left.\bar{b} \psi_{x x}\right|_{t_{f}}
\end{aligned}
$$

For $\tau \epsilon\left(\mathrm{t}_{2}, \mathrm{t}_{\mathrm{b}}\right]$ the dynamical equation is

$$
\left(\bar{x}+\delta_{x}\right)=f\left(\bar{x}+\delta_{x}, \bar{u}+\beta \sigma, \tau\right)
$$

and the cost functional is

$$
\mathrm{V}^{* k}\left(\overline{\mathrm{x}}+\delta_{\mathrm{x}}, \overline{\mathrm{b}}, \tau\right)=\int_{\tau}^{\mathrm{t} b} L\left(\overline{\mathrm{x}}+\delta_{\mathrm{x}}, \mathrm{t}\right) \mathrm{dt}+\overline{\mathrm{V}}^{*}\left(\overline{\mathrm{x}}+\delta_{\mathrm{x}}, \overline{\mathrm{b}}, \mathrm{t}_{\mathrm{b}}\right)
$$

$f$ It is easy to show that ${ }^{\prime}$ if the linear system $\delta_{\dot{x}}=f \delta_{x}+f_{u} \delta_{u}$ is completely controllable, and if $\psi_{\mathrm{x}}^{\mathrm{T}}\left(\overline{\mathrm{x}}, \mathrm{t}_{\mathrm{f}}\right)$ has full rank $\mathrm{s}$, the inverse $\mathrm{un}_{\mathrm{X}} \mathrm{Eq}$. (78) exists. 
Since the cost $\mathrm{V}^{*}\left(\overline{\mathrm{x}}+\delta_{\mathrm{x}}, \overline{\mathrm{b}}, \tau\right), \tau \in\left[\mathrm{t}_{2}, \mathrm{t}_{\mathrm{b}}\right]$ depends on $\sigma$, let us make this dependence explicit by defining

$$
J\left(\bar{x}+\delta_{x}, \bar{b}, \sigma, \tau\right) \equiv V^{*}\left(\bar{x}+\delta_{x}, \bar{b}, \tau\right)
$$

so that

$$
J\left(\bar{x}+\delta_{x}, \bar{b}, \sigma, \tau\right)=\int_{\tau}^{t_{b}} L\left(\bar{x}+\delta_{x}, t\right) d t+\bar{V}^{*}\left(\bar{x}+\delta_{x}, \bar{b}, t_{b}\right)
$$

In a similar way to that demonstrated in Section 5 , the following equations can be obtained,

$$
\begin{aligned}
& -\dot{J}_{x}=H_{x} \quad ; \quad J_{x}\left(t_{b}\right)=\bar{V}_{x}^{* k}\left(t_{b}\right) \\
& -\dot{J}_{x x}=H_{x x}+f_{x}^{T} J_{x x}+J_{x x} f_{x} ; J_{x x}\left(t_{b}\right)=\bar{V}_{x x}^{*}\left(t_{b}\right) \\
& -\dot{J}_{\sigma}=\beta^{T_{H_{u}}}=0 \quad ; \quad J_{\sigma}\left(t_{b}\right)=0 \\
& -\dot{J}_{x \sigma}=f_{x}^{T} J_{x \sigma}+\left(H_{x u}+J_{x x} f_{u}\right) \beta ; \quad J_{x \sigma}\left(t_{b}\right)=0 \\
& -\dot{J}_{\sigma \sigma}=J_{\sigma x} f_{u} \beta+f_{u}^{T}{ }^{T} J_{x \sigma} \quad ; \quad J_{\sigma \sigma}\left(t_{b}\right)=0
\end{aligned}
$$

where all quantities in (84) are evaluated at $\bar{x}, \bar{u}$. These equations can be integrated backwards from $t_{b}$ until $t_{2}$ is reached. At $t_{2}, \sigma$ is given by Eq. (78), and the expansion for $J\left(\bar{x}+\delta_{x}, \bar{b}, \sigma, t_{2}\right)$ to second-order in $\delta_{x}\left(t_{2}\right)$ and $\sigma$ is

$$
\begin{aligned}
J\left(\bar{x}+\delta_{x}, \bar{b}, \sigma, t_{2}\right)= & J\left(\bar{x}, \bar{b}, 0, t_{2}\right)+\left\langle J_{x}, \delta_{x}\right\rangle+\left\langle J_{\sigma}, \sigma\right\rangle+\left\langle\delta_{x}, J_{x \sigma} \sigma\right\rangle \\
& +\frac{1}{2}\left\langle\delta_{x}, J_{x x} \delta_{x}\right\rangle+\frac{1}{2}\left\langle\sigma, J_{\sigma \sigma} \sigma\right\rangle .
\end{aligned}
$$

Substituting into (84) the value of $\sigma$, we obtain

$$
\begin{aligned}
\mathrm{J}\left(\overline{\mathrm{x}}+\delta_{\mathrm{x}}, \overline{\mathrm{b}}, \gamma \delta_{\mathrm{x}}, \mathrm{t}_{2}\right)=\mathrm{J}\left(\overline{\mathrm{x}}, \overline{\mathrm{b}}, 0, \mathrm{t}_{2}\right)+\left\langle\mathrm{J}_{\mathrm{x}}, \delta_{\mathrm{x}}\right\rangle+\left\langle\mathrm{J}_{\sigma}, \gamma \delta_{\mathrm{x}}\right\rangle+\left\langle\delta_{\mathrm{x}}, \mathrm{J}_{\mathrm{x} \sigma} \gamma \delta_{\mathrm{x}}\right\rangle \\
+\frac{1}{2}\left\langle\delta_{\mathrm{x}}, \mathrm{J}_{\mathrm{xx}} \delta_{\mathrm{x}}\right\rangle+\frac{1}{2}\left\langle\delta_{\mathrm{x}}, \gamma^{\mathrm{T}_{\mathrm{J}}}{ }_{\sigma \sigma_{\mathrm{x}}}\right\rangle \delta
\end{aligned}
$$

Renaming the left-hand side of (85) as $\hat{J}\left(\bar{x}+\delta_{x}, \bar{b}, t_{2}\right)$ we obtain 


$$
\hat{J}_{x x}=J_{x x}+\gamma^{T} J_{\sigma \sigma} \gamma+J_{x \sigma} \gamma+\gamma^{T} J_{\sigma x}
$$

and

$$
\hat{\mathrm{J}}_{\mathrm{x}}=\mathrm{J}_{\mathrm{x}} \quad, \quad \text { since } \mathrm{J}_{\sigma}=0
$$

Equations (86) and (87) are the second and first partial derivatives of the cost at $t=t_{2}$, given that the terminal constraints (4) are satisfied to first-order.

From Section 5, the change in cost, owing to the presence of the rectangular pulse in the interval $\left[\mathrm{t}_{1}, \mathrm{t}_{2}\right]$ is

$$
\frac{1}{2} f_{u}^{T}\left(\bar{x}, t_{2}\right)\left[H_{x u}\left(\bar{x}, \hat{J}_{x}, t_{2}\right)+\hat{J}_{x x} f\left(\bar{x}, t_{2}\right)\right] \eta^{2} T^{2}+\text { higher-order term } s
$$

Thus the new necessary condition of optimality for singular problems with te rminal constraints is

$$
f_{u}^{T}\left(\bar{x}, t_{2}\right)\left[H_{x u}\left(\bar{x}, \hat{J}_{x}, t_{2}\right)+\hat{J}_{x x}^{f} f_{u}\left(\bar{x}, t_{2}\right)\right] \geqslant 0 .
$$

As mentioned earlier, the control $\beta(\tau) \sigma$ only ensures that the terminal constraints are satisfied to first-order. In the Appendix it is demonstrated that if the terminal constraints are satisfied to second-order, conclusion (89) is unaffected. This is true also if the terminal constraints are satisfied to higher-order, or satisfied exactly.

\section{Example}

Consider the following scalar control problem:

$$
\begin{aligned}
& \dot{x}=u \quad, \quad x(0)=1 \\
& V(1,0)=\int_{0}^{2} x^{2} d t-S x^{2}(2)
\end{aligned}
$$

with the terminal constraint that

$$
x(2)=0
$$


and the control constraint

$$
|u| \leqslant 1
$$

In Section 6 it was demonstrated that, in the absence of equality (93), and for $S>0$, the following control program is a stationary, non-minimizing solution:

$$
\begin{array}{lll}
u(\tau)=-1 & ; & \tau \in[0,1] \\
u(\tau)=0 & ; & \tau \in(1,2]
\end{array}
$$

We shall demonstrate now that with equality (93) present, the new necessary condition (89) is satisfied (by inspection the control program (95) is optimal for all S).

Since the singular arc extends from $t=1$ to $t=2$, we have $t_{b}=t_{f}=2$. For the above problem Eqs. (84) become

$$
\begin{aligned}
& -\dot{J}_{x}=2 x \\
& -\dot{J}_{x x}=2 \\
& -\dot{J}_{\sigma}=0 \\
& -\dot{J}_{x \sigma}=J_{x x} \\
& -\dot{J}_{\sigma \sigma}=2 J J_{x}
\end{aligned}
$$

Boundary conditions for Eqs. (96) are zero at $t=2$, except for

$$
J_{\mathrm{xx}}(2)=-\mathrm{S}
$$

From (96) and (97), we obtain the solutions

$$
\begin{aligned}
& J_{x}(\tau)=0 \\
& J_{X x}(\tau)=-S+2 \tau \\
& J_{\sigma}(\tau)=0 \\
& J_{x \sigma}(\tau)=-S \tau+\tau^{2} \\
& J_{\sigma \sigma}(\tau)=-S \tau^{2}+\frac{2}{3} \tau^{3}
\end{aligned}
$$

along the singular arc. 
In addition;

$$
\gamma=-\tau^{-1}
$$

where

$$
\tau=2-t, t \geqslant 1 .
$$

From (86), (98) and (99),

$$
\hat{\mathrm{J}}_{\mathrm{Xx}}=-\mathrm{S}+2 \tau+2\left(-\mathrm{S} \tau+\tau^{2}\right)\left(-\tau^{-1}\right)+\left(\tau^{-1}\right)^{2}\left(-\mathrm{S} \tau^{2}+\frac{2}{3} \tau^{3}\right)
$$

and we have that

$$
f_{u}^{T}\left(H_{x u}+\hat{J}_{x x^{\prime}} f_{u}\right)=\hat{J}_{x x}=\frac{2}{3} \tau
$$

so that the new necessary condition is satisfied for all $\tau \geqslant 0$, independent of $S$; this is the desired result.

\section{Generalized Legendre-Clebsch Necessary Condition}

In [23], Kelley et al used a special control variation of the form shown in Fig. 2 to derive the first generalization of the Legendre-Clebsch condition. They gave an heuristic argument to demonstrate that, if the control problem is normal, then a control variation can be found such that the terminal constraints (4) are met, at least to first-order, and the resulting change in cost owing to this added variation is negligible compared to that caused by the variation shown in Fig. 2 .

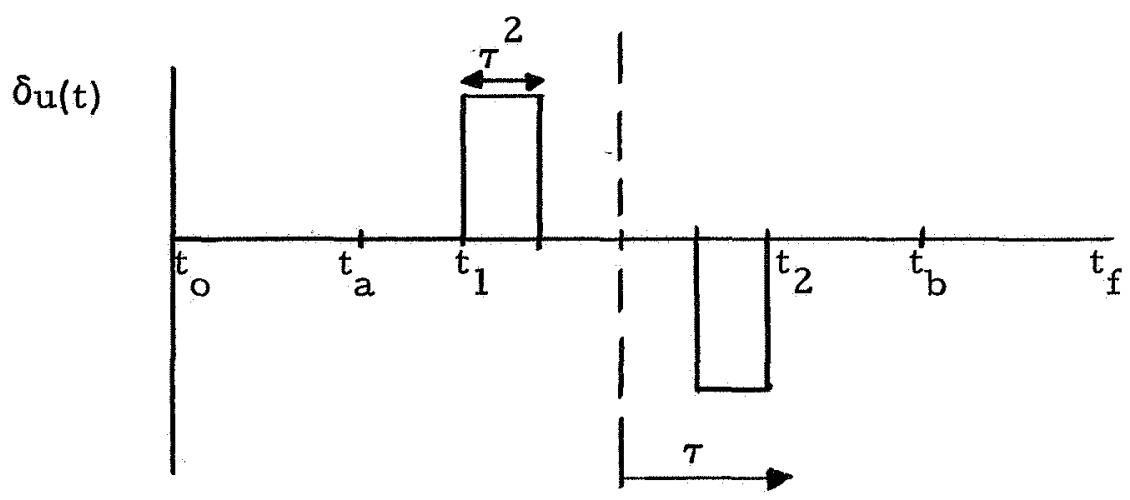

Figure 2 
If our rectangular pulse is replaced by Kelley's special variation, and if Eq. (68) is used to maintain the terminal equality (4) to first-order, then expansion of Eq. (21) yields -- upon requiring a(t $\left.t_{1}\right)$ to be greater than or equal to zero -- the first generalization of the Legendre-Clebsch condition:

$$
\frac{\partial}{\partial u}\left[\frac{d^{2}}{d t^{2}} H_{u}\left(\bar{x}, \hat{J}_{x}, t_{2}-\tau\right)\right]<0
$$

The normality assumption of Kelley and Robbins is the same as our assumption of controllability of

$$
\delta_{\dot{x}}=f_{x} \delta_{x}+f_{u} \delta_{u}
$$

and the maximal rank of

$$
\psi_{\mathrm{x}}^{\mathrm{T}}
$$

required to ensure the existence of the control variation

$$
\beta(\tau) \sigma
$$

which maintains satisfaction of terminal constraints (4) to first-order. The complete generalized Legendre-Clebsch condition can be derived by using Kelley's generalized special variation.

\section{Conclusion}

In this paper we have derived a new necessary condition of optimality for singular control problems. The control problem without terminal constraints was treated first. With terminal constraints present, a special admissible control variation has to be constructed; this requires that the control problem be normal.

+ Note that if the linearized system is completely controllable then condition (89) applies equally well to the control problem without terminal constraints; that is, (89) must be true for all matrices $\psi_{\mathrm{x}}^{\mathrm{T}}$ of full rank. 
The Differential Dynamic Programming technique was used to obtain an expression for the change in cost produced by the control variation. For the singular arc to be minimizing it is necessary that this change in cost be non-negative; from this requirement the new necessary conditions were deduced. Simple examples were used to illustrate the non-equivalence of the new conditions and the generalized Legendre-Clebsch condition. Finally, it was remarked that the generalized Legendre-Clebsch condition can be obtained by expanding Eq. (21) and using Kelley's special variation followed by the variation Eq. (68) which maintains satisfaction of the terminal constraints, Eq. (4), to first-order.

In some aerospace problems, stationary control functions have been determined which pass the generalized Legendre-Clebsch test, but whose optimality remains in doubt. The new necessary condition of optimality should prove useful in ascertaining whether indeed the se control functions are extremal or not.

Further, it is hoped that a useful sufficiency condition of optimality will evolve from the type of arguments presented in this paper, and that this will lead to the development of numerical techniques for solving singular optimal control problems. 
Acknowledgements

Stimulating discussions with Dr. J. L. Speyer and Professor

Y. C. Ho are gratefully acknowledged. 



\section{$\underline{\text { References }}$}

[1] Berkovitz, L. D., Variational Methods in Problems of Control and Programming, J. Math. Anal. Appl. , 3, No. 1, 1961, pp. 145-169.

[2] Dreyfus, S. E., Variational Problems with State Variable Inequality Constraints, J. Math. Anal. Appl., 4, 1962, pp. 297-308.

[3] Gamkrelidze, R. V., Optimal Processes with Bounded Phase Coordinates, Izv. Akad. Nauk (SSSR) Ser. Mat. 24, 1960, pp. 315-356.

[4] Chang, S. S. L., Optimal Control in Bounded State Space, Automatica, 1 , pp. 55-67.

[5] McIntyre, J., and Paiewonsky, B., On Optimal Control with Bounded State Variables, Advances in Control Systems (ed. C. T. Leondes), Vol. 5, Academic Press, New York, 1967.

[6] Speyer, J. L., Optimization and Control of Nonlinear Systems with Inflight Constraints, Ph.D. thesis, Harvard University, February 1968.

[7] Jacobson, D. H., Differential Dynamic Programming Methods for Solving Bang-Bang Control Problems, IEEE Trans. Auto. Control, AC-13, December 1968.

[8] Johnson, C. D., and Gibson, J. E., Singular Solutions in Problems of Optimal Control, IEEE Trans. Auto. Control, AC-8, 1963, pp. 4-15.

[9] Wonham, W. M., and Johnson, C. D., Optimal Bang-Bang Control with Quadratic Performance Index, ASME. Trans. J. Basic Eng., 86, 1964, pp. 107-115.

[10] Snow, D. R., Singular Optimal Controls for a Class of Minimum Effort Problems, J. SIAM Control, 2, 1964, pp. 203-219.

[11] Johnson, C. D., Singular Solutions in Optimal Control Problems, Advances in Control. Systems (C. T. Leondes, ed.), Vol. 2, Academic Press, New York, 1965.

[12] Athans, M., and Cannon, M. D., On the Fuel Optimal Singular Control of Nonlinear Second-Order Systems, IEEE Trans. Auto. Control, AC-9, 1964, pp. 360-370.

[13] Bass, R. W., and Weber, R. F., On Synthesis of Optimal Bang-Bang Feedback Control Systems with Quadratic Performance Criterion, Proc. 6th Joint Autom. Control Conf., Troy, New York, 1965, pp. 213-219.

[14] Hermes, H., Controllability and the Singular Problem, J. SIAM Control 2, 1964, pp. 241-260. 
[15] Hermes, H., and Haynes, G. W., On the Nonlinear Control Problem with Control Appearing Linearly, J. SIAM Control 1, 1963, pp. 85-107.

[16] Kelley, H. J., Singular Extremals in Lawden's Problem of Optimal Rocket Flight, J. AIAA, 1, 1963, pp. 1578-1580.

[17] Kelley, H. J., A Transformation Approach to Singular Subarcs in Optimal Trajectory and Control Problems, J. SIAM Control, 2, 1964, pp. 234-240.

[18] Robbins, H. M., Optimality of Intermediate-Thrust Arcs of Rocket Trajectories, J. AIAA, 3, 1965, pp. 1094-1098.

[19] Pfeiffer, C. G., Some New Results in Optimal Final Value Control Theory, J. Franklin Inst., 283, No. 5, 1967, pp. 404-425.

[20] Kelley, H. J., A Second Variation Test for Singular Extremals, J. AIAA, 2, 1964, pp. 1380-1382.

[21] Robbins, H. M., A Generalized Legendre-Clebsch Condition for the Singular Cases of Optimal Control, IBM, Federal Systems Division, Owego, New York, Report No. 66-825, p. 2043, 1966.

[22] Tait, K. S., Singular Problems in Optimal Control, Ph. D. thesis, Harvard University, 1965.

[23] Kelley, H. J., Kopp, R. E., Moyer, H. G., Singular Extremals, Topics in Optimization (G. Leitman, ed.), Academic Press, 1967.

[24] Jacobson, D. H., New Second-Order and First-Order Algorithms for Determining Optimal Control: A Differential Dynamic Programming Approach, J. Opt. Theory Appl., 2, No. 6, 1968.

[25] Jacobson, D. H., Second-Order and Second-Variation Methods for Determining Optimal Control: A Comparative Study Using Differential Dynamic Programming, Int. J. Control, 7, No. 2, 1968, pp. 175-196.

[26] Jacobson, D. H., and Mayne, D. Q., Differential Dynamic Programming, American Elsevier Publishing Co., New York, 1969, to appear. 
Appendix

\section{A. 1. Satisfaction of Terminal Constraints to Second-Order}

Expansion of equality $(4)$ to second-order in $\delta_{x}\left(t_{f}\right)$ about $\bar{x}\left(t_{f}\right)$ yields

$$
\psi_{x}\left(\bar{x}, t_{f}\right) \delta_{x}+\frac{1}{2} \psi_{x x}\left(\bar{x}, t_{f}\right) \delta_{x} \delta_{x}=0
$$

and the expansion of (1) to second-order in $\delta_{\mathrm{x}}$ and $\delta_{\mathrm{u}}$, about $\overline{\mathrm{x}}, \overline{\mathrm{u}}$ is

$$
\delta_{x}=f_{x} \delta_{x}+f_{u} \delta_{u}+f_{u x} \delta_{u} \delta_{x}+\frac{1}{2} f_{x x} \delta_{x} \delta_{x} ; \quad \delta_{x}\left(t_{2}\right) \neq 0
$$

The solution of $(A-2)$, correct to second-order terms is

$$
\begin{aligned}
\delta_{x}(t)=\Phi\left(t, t_{2}\right) \delta_{x}\left(t_{0}\right) & +\int_{t_{2}}^{t} \Phi(t, \tau) f_{u}(\tau) \delta_{u}(\tau) d \tau+\frac{1}{2} \Phi_{x}\left(t, t_{2}\right) \delta_{x}(t) \delta_{x}\left(t_{2}\right) \\
& +\frac{1}{2} \int_{t_{2}}^{t} \Phi(t, \tau) f_{u x}(\tau) \delta_{u}(\tau) \delta_{x}(\tau) d \tau+\frac{1}{2} \int_{t_{2}}^{t} \Phi_{x}(t, \tau) \delta_{x}(t) f_{u}(\tau) \delta_{u}(\tau) d \tau
\end{aligned}
$$

where

$$
\dot{\Phi}(t, \tau)=f_{x} \Phi(t, \tau) \quad ; \quad \Phi(\tau, \tau)=I
$$

and

$$
\dot{\Phi}_{X}(t, \tau)=f_{X} \Phi_{X}(t, \tau)-\Phi_{X}(t, \tau) f_{X}+f_{X X} \Phi(t ; \tau) ; \quad \Phi_{X}(\tau, \tau)=0 .
$$

The quadratic terms on the $r . h$. s. of $(A-3)$ contain $\delta x(t)$ : for $(A-3)$ to be correct to second-order terms, the following expression can be used for $\delta_{x}(t)$ in the r.h.s. of $(A-3)$ :

$$
\delta_{x}(t) \cong \Phi\left(t, t_{2}\right) \delta_{x}\left(t_{2}\right)+\int_{t_{2}}^{t} \Phi(t, \tau) f_{u}(\tau) \delta_{u}(\tau) d \tau
$$

$$
\psi_{x x}\left(\bar{x}, t_{f}\right) \delta_{x} \delta_{x} \equiv \sum_{i=1}^{n} \sum_{j=1}^{n} \psi_{x_{i} x_{j}} \delta_{x_{i}} \delta_{x_{j}} ; \text { similarly for } f_{u x} \delta_{u} \delta_{x} \text { and } f_{x x} \delta_{x} \delta_{x}
$$


Substituting $(A-6)$ into the r.h.s. of $(A-3)$, we obtain

$$
\begin{aligned}
\delta_{x}(t)=\Phi(t, & \left.t_{2}\right) \delta_{x}\left(t_{2}\right)+\int_{t_{2}}^{t} \Phi(t, \tau) f_{u}(\tau) \delta_{u}(\tau) d \tau \\
& +\frac{1}{2} \Phi_{x}\left(t, t_{2}\right)\left[\Phi\left(t, t_{2}\right) \delta_{x}\left(t_{0}\right)+\int_{t_{2}}^{t} \Phi(t, \tau) f_{u}(\tau) \delta_{u}(\tau) d \tau\right] \delta_{x}\left(t_{2}\right) \\
& +\frac{1}{2} \int_{t_{0}}^{t} \Phi(t, \tau) f_{u x}(\tau) \delta_{u}(\tau)\left[\int_{t_{2}}^{t} \Phi\left(\tau, t_{2}\right) \delta_{x}\left(t_{2}\right)\right. \\
& \left.+\int_{t_{2}}^{\tau} \Phi\left(\tau, \tau^{\prime}\right) f_{u}\left(\tau^{\prime}\right) \delta_{u}\left(\tau^{\prime}\right) d \tau^{\prime}\right] d \tau+\frac{1}{2} \int_{t_{2}}^{t} \Phi_{x}(t, \tau)\left[\Phi\left(t, t_{2}\right) \delta_{x}\left(t_{2}\right)\right. \\
& \left.+\int_{t_{2}}^{t} \Phi\left(t, \tau^{\prime}\right) f_{u}\left(\tau^{\prime}\right) \delta_{u}\left(\tau^{\prime}\right) d \tau^{\prime}\right] \delta_{u}(\tau) d \tau
\end{aligned}
$$

The form of $\delta u(\tau)$, based on satisfaction of the terminal constraints to first-order is, from (68),

$$
\delta_{u}(\tau)=\beta(\tau) \sigma
$$

where $\sigma$ is given by $(78)$ as

$$
\sigma=\gamma \delta_{x}\left(t_{2}\right)
$$

so that $\sigma$ is of first-order in $\delta_{x}\left(t_{2}\right)$.

Let us assume now that $\delta_{u}(\tau)$ is of the form

$$
\delta \mathrm{u}(\tau)=\beta(\tau)[\sigma+a]
$$

where $a$ is of order $\delta_{x}{ }^{2}\left(t_{2}\right)$. Then, to second-order in $\delta_{x}\left(t_{2}\right), \delta_{x}\left(t_{f}\right)$ is: 


$$
\begin{aligned}
& \delta_{x}\left(t_{f}\right)=\Phi\left(t_{f}, t_{2}\right) \delta_{x}\left(t_{2}\right)+\int_{t_{2}}^{t_{f}} \Phi\left(t_{f}, \tau\right) f_{u}(\tau) \beta(\tau)[\sigma+a] d \tau \\
&+\frac{1}{2} \Phi_{x}\left(t_{f}, t_{2}\right)\left[\Phi\left(t_{f}, t_{2}\right) \delta_{x}\left(t_{0}\right)+\int_{t_{2}}^{t_{f}} \Phi\left(t_{f}, \tau\right) f_{u}(\tau) \beta(\tau) \sigma d \tau\right] \delta_{x}\left(t_{2}\right) \\
&+\frac{1}{2} \int_{t_{o}}^{t_{f}} \Phi\left(t_{f}, \tau\right) f_{u x}(\tau) \beta(\tau) \sigma\left[\int_{t}^{\tau}\left(\tau, t_{2}\right) \delta_{x}\left(t_{2}\right)\right. \\
&\left.+\int_{t_{0}}^{\tau} \Phi\left(\tau, \tau^{\prime}\right) f_{u}\left(\tau^{\prime}\right) \beta\left(\tau^{\prime}\right) \sigma d \tau^{\prime}\right] d \tau+\frac{1}{2} \int_{t_{2}}^{t_{f}} \Phi_{x}\left(t_{f}, \tau\right)\left[\Phi\left(t_{f}, t_{2}\right) \delta_{x}\left(t_{2}\right)\right. \\
&\left.+\int_{t_{2}}^{t_{f}} \Phi\left(t_{f^{\prime}}, \tau^{\prime}\right) f_{u}\left(\tau^{\prime}\right) \beta\left(\tau^{\prime}\right) \sigma d \tau^{\prime}\right] \beta(\tau) \sigma d \tau
\end{aligned}
$$

If $(A-11)$ is now substituted into $(A-1)$, then the first-order terms in $\sigma$ vanish, because of (73), leaving

$$
\left[\int_{t_{2}}^{t_{f}} \psi_{x}\left(\bar{x}, t_{f}\right) f_{u}(\tau) \beta(\tau) d \tau\right] a+\text { terms of order } \delta_{x}{ }^{2}\left(t_{2}\right)=0
$$

The quantity in the square brackets on the $1 . \mathrm{h} . \mathrm{s}$. of $(A-12)$ is, from (76), just

$$
\psi_{x}\left(\bar{x}, t_{f}\right) W\left(t_{2}, t_{b}\right) \psi_{x}^{T}\left(\bar{x}, t_{f}\right)
$$

which is invertible. So, from $(A-12)$, a can be found and it is of order $\delta_{x}^{2}\left(t_{2}\right)$. Thus a control variation of form

$$
\delta \mathrm{u}(\tau)=\beta(\tau)[\sigma+\mathrm{a}]
$$

where $\sigma$ is first-order in $\delta_{x}\left(t_{2}\right)$ and $a$ is second-order in $\delta_{x}\left(t_{2}\right)$ maintains the terminal equality (4) correct to second-order terms. Now if $(\mathrm{A}-14)$ is substituted into (85), we find that, because a is second-order in $\delta_{x}\left(t_{2}\right)$, and $J_{\sigma}=0,(86)$ and (87) do not contain $a$; thus the conclusion (89) is unaffected if we satisfy the terminal constraints to second-order rather 
than to first-order. Satisfaction of terminal constraints to higher-order yields the same result. This confirms that, in order to include all second-order terms in an expansion of the cost functional, it is only necessary to expand the Hamiltonian to second-order terms and the dynamic and terminal constraints to first-order terms. 


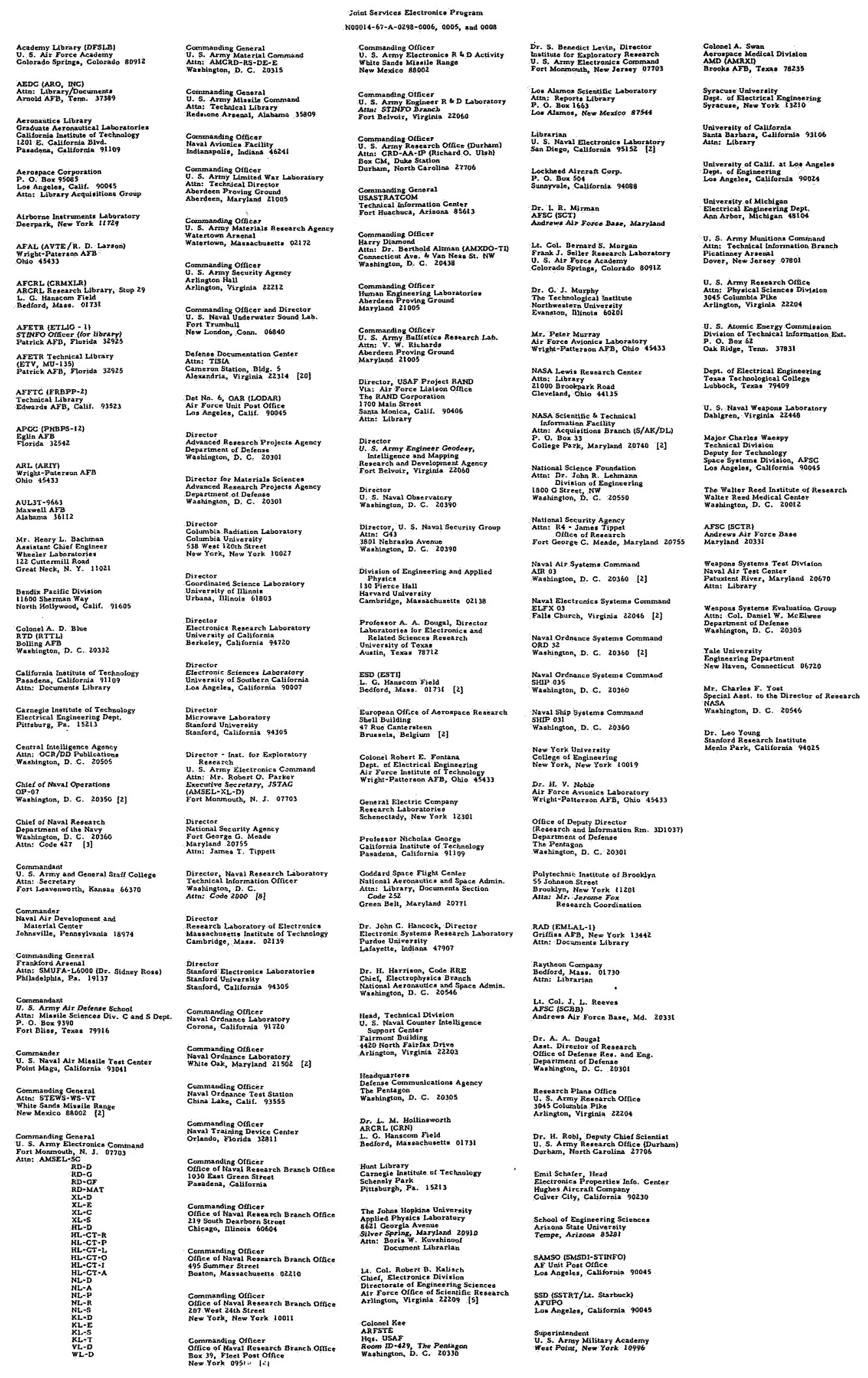


DOCUMENT CONTROL DATA - R \& D

(Security classification of title, body of abstract and indexing annotation must be entered when the overall report is classified) 1. ORIGINATING ACTIVITY (Corporate author)

Division of Engineering and Applied Physics

Harvard University

Cambridge, Massachusetts

3. REPORT TITLE

A NEW NECESSARY CONDITION OF OPTIMALITY FOR SINGULAR CONTROL PROBLEMS

4. DESCRIPTIVE NOTES (Type of report and, inclusive dates)

Interim technical report

5. AU THOR(S) (First name, middle initial, last name)

D. H. Jacobson

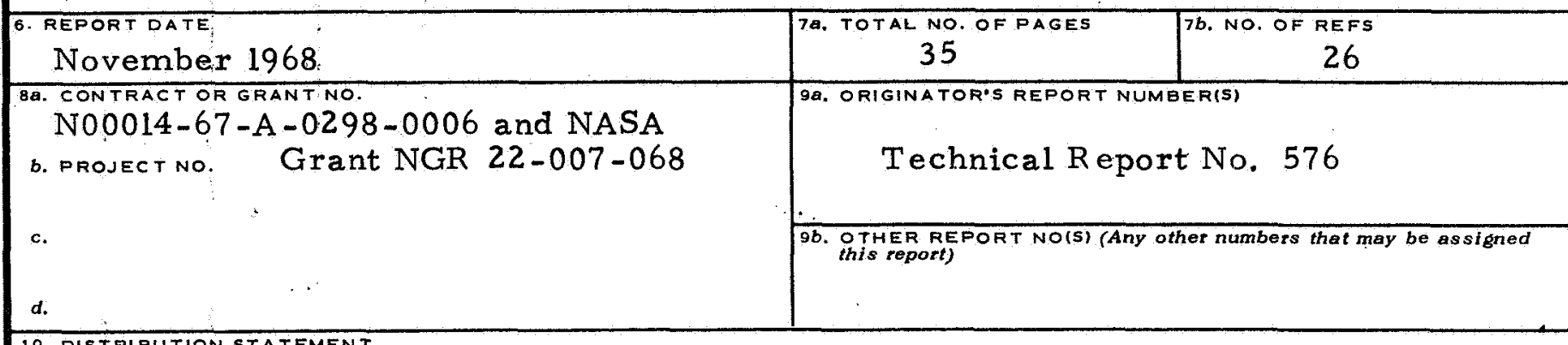

10. DISTRIBUTION STATEMENT

This document has been approved for public release and sale; its distribution is unlimited. Reproduction in whole or in part is permitted by the U. S. Government.

\begin{tabular}{l|l|l} 
11. SUPPLEMENTARY NOTES & 12. SPONSORING MILITARY ACTIVITY
\end{tabular}

Office of Naval Research

13. ABSTRACT

A variation in the form of a rectangular pulse of short duration, is introduced into the singular control function. The technique of Differential Dynamic Programming is used to obtain an expression for the change in cost produced by the control variation, and a new necessary condition of optimality is deduced by requiring that this change in cost be non-negative. When terminal equality constraints are present the control variation takes the form of a rectangular pulse followed by a 'special variation' which is chosen to keep the terminal equality constraints satisfied to first-order simple control problems are used to illustrate the non-equivalence of the new necessary condition and the generalized Legendre-Clebsch condition. 


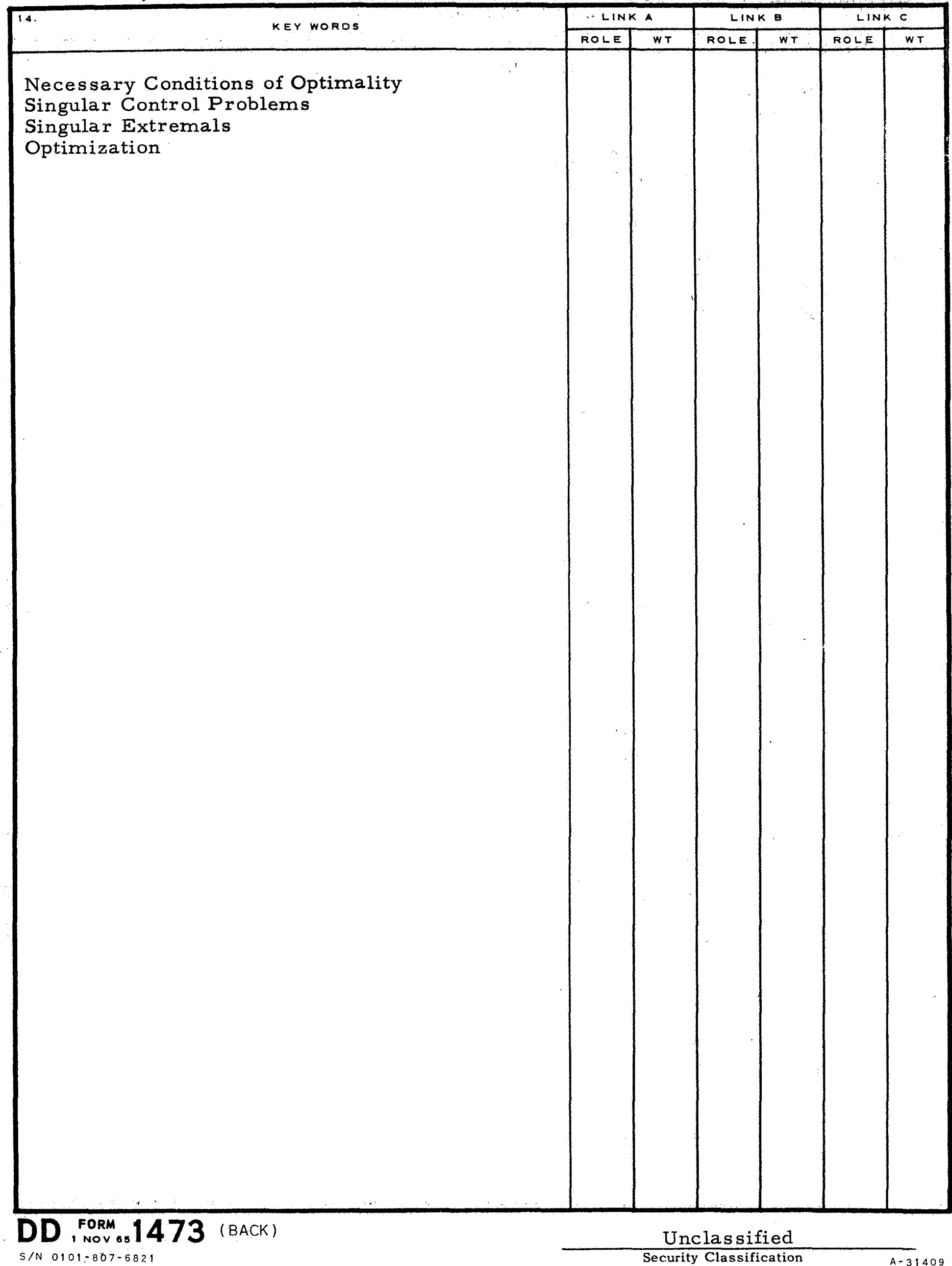

